\title{
Viewpoint
}

\section{Patient Selection and Assessment Recommendations for Deep Brain Stimulation in Tourette Syndrome}

\author{
Jonathan W. Mink, MD, PhD, ${ }^{1 *}$ John Walkup, MD, ${ }^{2}$ Kirk A. Frey, MD, PhD, ${ }^{3}$ Peter Como, $\mathrm{PhD}^{1}$ \\ Danielle Cath, MD, PhD,${ }^{4}$ Mahlon R. DeLong, MD, ${ }^{5}$ Gerald Erenberg, MD ${ }^{6}$ Joseph Jankovic, MD, ${ }^{7}$ \\ Jorge Juncos, MD, ${ }^{5}$ James F. Leckman, MD, ${ }^{8}$ Neal Swerdlow, MD, PhD, ${ }^{9}$ \\ Veerle Visser-Vandewalle, MD, $\mathrm{PhD},{ }^{10}$ Jerrold L. Vitek, MD, $\mathrm{PhD},{ }^{6}$ \\ and the Tourette Syndrome Association, Inc.
}

\begin{abstract}
${ }^{I}$ Department of Neurology, University of Rochester, Rochester, New York, USA; ${ }^{2}$ Department of Psychiatry, Johns Hopkins University, Baltimore, Maryland, USA; ${ }^{3}$ Department of Radiology, University of Michigan, Ann Arbor, Michigan, USA; ${ }^{4}$ Department of Psychiatry, The Netherlands; ${ }^{5}$ Department of Neurology, Emory University, Atlanta, Georgia, USA; ${ }^{6}$ Department of Neurology, Cleveland Clinic, Cleveland, Ohio, USA; ${ }^{7}$ Department of Neurology, Baylor College of Medicine, Houston, Texas, USA; ${ }^{8}$ Child Study Center, Yale University, New Haven, Connecticut, USA; ${ }^{9}$ Department of Psychiatry, University of California San Diego, San Diego, California, USA; ${ }^{10}$ Department of Neurosurgery, European Graduate School of Neuroscience (EURON), University Hospital Maastricht, Maastricht, The Netherlands
\end{abstract}

\begin{abstract}
In response to recent publicity regarding the potential use of deep brain stimulation (DBS) for reducing tic severity in Tourette's syndrome (TS), the Tourette Syndrome Association convened a group of TS and DBS experts to develop recommendations to guide the early use and potential clinical trials of DBS for TS and other tic disorders. The goals of these recommendations are to ensure that all surgical candidates are (1) fully informed about the risks, benefits, and alternative
\end{abstract}

treatments available; (2) receive a comprehensive evaluation before surgery to ensure that DBS is clearly the appropriate clinical treatment choice; and (3) that early clinical experience will be documented publicly to facilitate rational decisionmaking for both clinical care and future clinical trials. (C) 2006 Movement Disorder Society

Key words: deep brain stimulation; Tourette syndrome; tics; treatment
We recommend that surgical candidates undergo a comprehensive preoperative assessment by experts in Tourette's syndrome (TS) and comorbid psychiatric disorders to ensure that candidates meet rigorous inclusion and exclusion criteria, that evaluations use standardized rating scales, and that electrode placement is carefully documented. We further recommend complete comprehensive postoperative assessments, including neurologi-

*Correspondence to: Dr. Jonathan W. Mink, 601 Elmwood Avenue, POB 631, Rochester, NY 14642.

E-mail: jonathan_mink@urmc.rochester.edu

Received 26 July 2005; Revised 27 February 2006; Accepted 23 March 2006

Published online 21 September 2006 in Wiley InterScience (www. interscience.wiley.com). DOI: 10.1002/mds.21039 cal, psychiatric, and neuropsychological evaluations. When publishing the results of these early efforts, each investigating site should use the same anatomical nomenclature, assessment protocols, programming strategies, and outcome instruments so that results can be compared across sites. These goals require meticulous screening, outstanding surgical skills, randomization, suitable control conditions, and uniform data collection. These recommendations are consistent with those offered previously for evaluating surgical treatments in Parkinson's disease and Huntington's disease.

Deep brain stimulation (DBS) has proven useful for treatment of several movement disorders, including Parkinson's disease, essential tremor, and dystonia. ${ }^{1-3}$ It is currently under investigation for treatment of several 
other conditions including epilepsy, ${ }^{4}$ obsessive-compulsive disorder (OCD), ${ }^{5}$ and Tourette's syndrome or tics. ${ }^{6-10}$ The substantial publicity regarding the apparent successful treatment of TS with DBS has increased interest among individuals with TS, their families, and the clinicians who care for them. As a patient and family support agency, the Tourette Syndrome Association (TSA) has an interest in ensuring that people with TS and tic disorders and their clinicians receive the latest information regarding new and potentially helpful treatments, but also that new and experimental treatments are evaluated in rigorous protocols that can provide critical information on the safety and efficacy of these therapies. As scientists who care for people with TS, we are well aware of how frequently early case reports and open case series have offered initial hope only for subsequent controlled trials to fail to demonstrate efficacy or reveal significant safety concerns. Given the great potential for benefit as well as harm, the TSA convened a group of internationally recognized experts on TS and DBS to develop recommendations to guide early investigations and use of DBS in tic disorders.

The assessment of tic severity for the purposes of conducting investigations of DBS is challenging given the complexity of the clinical features, the range of severity, and the waxing and waning course of tic symptoms. ${ }^{11}$ Because of the waxing and waning course, it is difficult to interpret the outcome of clinical trials that do not use randomization and suitable comparison conditions or controls. Based on the efficacy of DBS for other disorders, and early published reports on a small number of TS patients, we believe that proceeding with investigations of DBS is justified. We recommend that these studies be pursued with caution to obtain data that will guide future decisions on the role of DBS in the treatment of TS. Our proposal has the following goals: (1) to provide defensible inclusion and exclusion criteria; (2) to recommend valid and reliable outcome measures; (3) to stress the importance of collecting comparable data across centers; (4) to advocate on behalf of the TS community by the establishing accountability standards that outline the minimum effort necessary to ensure the safety of those undergoing the procedure now and in the future. To achieve these goals will require meticulous screening, outstanding surgical skills, randomization, suitable control conditions, and uniform data collection of data. The assessment protocols and patient selection criteria detailed here are consistent with those offered previously to evaluate surgical treatments in Parkinson's disease ${ }^{12}$ and Huntington's disease. ${ }^{13}$ Below is basic background information on TS, the implications that the phenomenology and natural history of TS may have on the evaluation of DBS, basic inclusion and exclusion criteria, and recommendations regarding selecting and documenting neurosurgical targets, perioperative imaging, and assessment of results in this population.

\section{CLINICAL FEATURES AND NATURAL HISTORY OF TS}

The defining symptoms of TS are motor and phonic (vocal) tics. ${ }^{14}$ Motor tics are stereotyped repetitive involuntary movements that typically involve the face, head, and upper body. Phonic (vocal) tics are sounds such as sniffing, grunting, or barking that are associated with muscle contractions of the oropharynx and diaphragm. Tic severity is based on the frequency, intensity, and complexity of movements and sounds and can range from simple and infrequent to complex, intense, and nearly continuous. Tics may also change their anatomical location, pattern, severity, and complexity over time. Tics are influenced by environmental factors: stressful and exciting activities are associated with transient increases in tic severity, and relaxation and calm, focused activities are associated with transient reduction of tic severity. Also, like many other medical conditions, psychological factors may affect the symptom expression or clinical presentation. For example, in extreme cases such as the ones who might be considered for surgery, there are reports of patients who exaggerate existing symptoms or generate factitious tic-like symptoms..$^{15-17}$

Tics most often begin in the first decade of life, usually between 5 and 7 years of age. They wax and wane, with peak severity in early adolescence with a gradual decrease in severity into adulthood. ${ }^{18,19}$ Remission of tics may occur in the third decade of life in up to $50 \%$ of patients, but to date, there are no prognostic features that predict which patients will have a remission in their symptoms. However, a small percentage of patients have severe, disabling tics refractory to standard medical treatment that continue or even increase in adulthood.

\section{IMPACT OF THE CLINICAL FEATURES AND NATURAL HISTORY OF TS ON DBS TREATMENT}

Given the fluid characteristics of tics over time and the role of environmental and psychological factors in attenuating or exacerbating tic symptoms, any clinical trial of DBS for TS must use sound clinical trial methods (e.g., adequate baseline observation periods, randomization, and suitable control conditions). Suitable candidates for DBS will be adults who have been evaluated comprehensively and who have undergone standard medical therapy for their tic disorder but who continue to experience severe tics. Those with an overall mild-moderate 
course during childhood and adolescence and a severe exacerbation in adulthood are outliers ${ }^{18}$ and may not be suitable candidates for this experimental procedure. Those going through significant but transient psychosocial or other stressors are also not good candidates. In our experience, patients cannot always be relied upon to report such stressors. Interviews with secondary informants (i.e., close family members) are important to identify these potential stressors. Also, given the potential complexity of TS symptoms, it may be difficult to assess intraoperatively the appropriate electrode placement resulting in suboptimal outcome. For example intraoperative assessment may be difficult for patients with selfinjurious tics or prominent head and neck tics.

\section{COMORBID SYMPTOMS IN TS}

Although tics are the defining symptoms in TS, many individuals with TS have other symptoms, including OCB, attention deficit hyperactivity disorder (ADHD), anxiety, and mood disorders. ${ }^{20} \mathrm{Up}$ to $50 \%$ of patients with TS have OCB. Similarly, up to 50\% of people with TS have ADHD symptoms. Smaller, but significant, percentages have anxiety, depression, or other affective symptoms. Identification of these comorbid symptoms is critical as they are often more impairing than the tics themselves. The diversity of these comorbid symptoms, and their potentially aggravating effects on tic severity and overall disability, further complicate the planning and interpretation of treatment trials that focus on interventions to reduce tic severity. In addition to comorbid symptoms, some with TS may also have significant psychosocial problems. Psychosocial difficulties may be associated with significant impairment that needs to be accounted for in the patients' preoperative assessment.

\section{IMPLICATION OF COMORBID SYMPTOMS IN TS ON DBS TREATMENT}

It is not known whether DBS for tics will have a beneficial, neutral, or deleterious effect on comorbid symptoms. In published case reports, DBS of the medial thalamus appears to have had a beneficial effect on some comorbid symptoms ${ }^{10}$; however, investigators and clinicians should be prepared for the possibility of divergent results. A complete baseline assessment of comorbid symptoms and psychosocial functioning is required before DBS surgery. The goals of these assessments are to ensure that the (1) subjects selected have chronically severe tics that account for their disability, (2) comorbid conditions have been adequately treated and do not account for significant disability, and (3) subjects can undergo the procedure safely and actively participate in their postoperative care, that is, adhere to treatment schedules and recommendations and to participate in the outcome assessment of their symptoms.

\section{INCLUSION/EXCLUSION CRITERIA}

The following inclusion and exclusion criteria have been developed to identify ideal candidates for DBS. It is likely that, as more reports are published, these criteria may need to be re-evaluated. At this time, ideal candidates will have severe and impairing tics and have failed exhaustive medical and behavioral treatment options. They should have no medical, neurological, or psychiatric conditions that increase the risk of the procedure or preclude full participation in the procedure, postprocedural care, or compromise the accurate assessment of the outcomes. Candidates must also be fully prepared for the possibility that the procedure will not work or may have deleterious effects, and have the personal resiliency and social support to live with such a negative outcome.

Eligible subjects should have received and failed to respond to optimal and systematic treatment for their tics and comorbid conditions before consideration of surgery. Psychological assessment should be done to determine suitability as a surgical candidate. Assessment and treatment of psychosocial factors need to be undertaken to ensure that the candidate can fully participate in his or her postoperative care and outcome assessment.

Candidates for DBS in TS should be evaluated by a multidisciplinary team, including a neurologist, a psychiatrist, and a doctoral level psychologist or neuropsychologist with expertise in TS and comorbid conditions. The team should explore psychopathologies or psychosocial factors that may be contributing to symptom severity or overall impairment. There should be a detailed assessment of the treatment history to establish adequacy of treatment trials as per current treatment standards. ${ }^{11,20-23}$ The treatment history should include attempts at managing these conditions with behavioral interventions..$^{24,25}$ The time for surgery is not during periods of acute or subacute, but self-limited psychosocial stress.

\section{Inclusion Criteria}

1. The patient must be at least 25 years old (with rare potential exceptions). Although this criterion is somewhat arbitrary, the goal is to ensure that included individuals have a stable degree of severity in adulthood that predicts a low probability of spontaneous improvement of tics consistent with the natural course of illness.

2. The patient should have a chronic and severe tic disorder with severe functional impairment. There should be a standardized videotape assessment documenting tic severity ${ }^{26,27}$ and a Yale Global Tic Severity Scale $\left(\right.$ YGTSS) ${ }^{28}$ total tic severity score $>35 / 50$ for at 
least 12 months. A YGTSS total tics score threshold of 35 falls in the middle of the "marked" severity range, ${ }^{29-38}$ indicating "tics frequent and quite noticeable in most situations most of the time" and includes patients with "severe" tics, ${ }^{39-49}$ indicating "tics associated with a significant impairment in their primary social role such that functioning in usual settings was impossible or placed in serious jeopardy." 18 The threshold was chosen to ensure that subjects undergoing DBS have marked to severe tics.

3. The patient must have failed conventional medical therapy for tics. The patient should have failed treatment trials (lack of efficacy or severe side effects) of adequate dose and duration of medication from three different pharmacological classes. Specifically, this medication should include an alpha-adrenergic agonist, two dopamine antagonists (typical and atypical), and a benzodiazepine.

4. The patient should have been evaluated for the suitability, and implementation if suitable, of behavioral interventions to reduce tic severity or the severity of comorbid symptoms. Although behavioral treatments for tics are themselves investigational at this time, the relatively low risk and potential for benefit in some patients may make a trial of such treatment before surgery a reasonable precursor to DBS treatment.

5. The patient must have received stable and optimized treatment of comorbid medical, neurological, and psychiatric disorders for the past 6 months.

6 . The patient must be actively involved and compliant with psychological interventions to address ongoing psychosocial problems. These interventions must have been in place at for least 6 months.

\section{Exclusion Criteria}

1. The patient must not have a tic disorder or other movement disorder problem attributable to another medical, neurological, or psychiatric disorder.

2. The patient must not have severe medical, neurological, psychiatric, or cognitive disorders that inordinately increase the risk of a failed procedure, surgical complications, or impede recovery and assessment of outcome (e.g., severe cardiovascular, pulmonary, or hematological disorders, epilepsy, history of head injury, stroke, neurodegenerative disease, autism, schizophrenia, bipolar disorder, recent substance dependence).

3. The patient must not be likely to benefit from psychological intervention (with an experienced clinician) for tic suppression.

4. The patient must not have significant psychosocial factors that increase the risk of the procedure, or complicate the recovery period and assessment of outcome.
For example, a history of noncompliance with previous medical and psychosocial treatments efforts, multiple failed medication treatments of inadequate dose or duration, a history of multiple other surgical procedures with poor outcome, unexplained gaps in the medical history, or pending lawsuits or other legal action.

5. The patient must not be unwilling to be involved in ongoing treatment for psychosocial problems or risk factors.

\section{IDENTIFICATION OF THE APPROPRIATE ANATOMICAL TARGET}

Stereotaxic neurosurgical treatment of any neurologic or psychiatric condition requires meticulous target selection. The modern success of lesioning and DBS in the treatment of Parkinson's disease has resulted from the availability of a good animal model, a testable neuronal circuit model based on a large body of data, and an adequate understanding of the anatomy and physiology of basal ganglia circuits underlying Parkinson's disease. Unfortunately, there is no good animal model of TS, the underlying neuronal circuitry is not well understood, and the physiology is not known. There is a large body of data suggesting abnormal basal ganglia function in TS, but little consensus on which parts of the basal ganglia or their extensive connections with cerebral cortex are responsible for tics. ${ }^{29-33,50}$ Similarly, there is little specific information on the neuronal circuitry responsible for comorbid symptoms commonly associated with TS. The medical literature contains several reports of stereotaxic lesions for treatment of tics with scant outcome data and questionable target localization in many cases. ${ }^{9}$ There have been a few reports of DBS for TS. ${ }^{6,8,9}$ The centromedian-parafascicular complex (CM-PF) of the thalamus has been targeted bilaterally in the majority of those cases, ${ }^{8,10}$ but the internal segment of the globus pallidus (GPi), ${ }^{6,8}$ and the anterior limb of the internal capsule ${ }^{7}$ have also been targeted. To date, 6 cases of DBS for TS have been published (Table 1). All have involved bilateral implantation and stimulation. At this time, there are insufficient data to recommend one site over the others.

It is essential that any investigation of DBS for TS include accurate postoperative imaging to identify the actual electrode placement. Even in the hands of experienced surgeons, there can be a difference of several critical millimeters between the intended target and the final electrode placement. In a region such as the medial thalamus that encompasses many small nuclei, the localization is especially important. It is also important in $\mathrm{GPi}$, a large structure that includes multiple circuits with different functions. ${ }^{34}$ Final target location cannot be assumed from preoperative imaging. Postoperative imag- 
TABLE 1. Summary of published reports of DBS for TS

\begin{tabular}{|c|c|c|c|c|c|c|}
\hline Age/sex & $\begin{array}{l}\text { Length of } \\
\text { follow-up }\end{array}$ & Comorbidity & $\begin{array}{c}\text { DBS } \\
\text { site }\end{array}$ & $\begin{array}{l}\text { Baseline } \\
\text { YGTSS }\end{array}$ & Post-op YGTSS & Reference \\
\hline \multirow[t]{2}{*}{$36 / \mathrm{F}^{\mathrm{a}}$} & 24 mos & $\begin{array}{l}\text { Anxiety, depression, borderline } \\
\text { personality }\end{array}$ & GPi & $84 / 100$ & $29 / 100$ & (8) \\
\hline & & & CM-PF & $84 / 100$ & $30 / 100$ & \\
\hline $27 / M$ & $14 \mathrm{mos}$ & Depression & GPi & $83 / 100$ & $44 / 100$ & (6) \\
\hline $42 / \mathrm{M}$ & $5 \mathrm{yr}$ & OCD & CM-PF & N.R. & $\begin{array}{l}\text { N.R.; video tic count } \\
\text { decreased } 90 \%\end{array}$ & (10) \\
\hline 28/M & $1 \mathrm{yr}$ & OCD & CM-PF & N.R. & $\begin{array}{l}\text { N.R.; video tic count } \\
\text { decreased } 72 \%\end{array}$ & (10) \\
\hline $45 / \mathrm{M}$ & $8 \operatorname{mos}$ & Possible OCD & CM-PF & N.R. & $\begin{array}{l}\text { N.R.; video tic count } \\
\text { decreased } 83 \%\end{array}$ & (10) \\
\hline $37 / \mathrm{F}$ & $18 \mathrm{mos}$ & None & AIC & N.R. & $20 \%$ improved & (7) \\
\hline
\end{tabular}

${ }^{\text {a } E l e c t r o d e s ~ w e r e ~ i m p l a n t e d ~ b i l a t e r a l l y ~ i n t o ~ b o t h ~ G P i ~ a n d ~ C M-P F ~ i n ~ t h i s ~ p a t i e n t . ~ S t i m u l a t i o n ~ o f ~ b o t h ~ G P i ~ a n d ~ C M-P F ~ d i d ~ n o t ~ p r o v i d e ~ a d d i t i o n a l ~}$ benefit.

DBS, deep brain stimulation; TS, Tourette's syndrome; AIC, anterior limb of internal capsule; CM-PF, centromedian-parafascicular complex; GPi, globus pallidus pars interna; N.R., not reported; OCD, obsessive-compulsive disorder; YGTSS, Yale Global Tic Severity Scale.

ing is essential. This imaging should be optimized to allow visualization of the target structure while minimizing the artifact associated with the DBS lead to allow one to determine the location of the lead within the target structure.

\section{Neuroimaging Recommendations}

It is necessary to identify accurately the electrode lead location(s) achieved to evaluate outcomes from DBS and to facilitate pooling of anatomical data across institutions. ${ }^{35}$ To this end, it is recommended that a standardized neuroimaging mapping be used pre- and postoperatively with equal effort placed on establishing an "optimal" therapeutic DBS electrode configuration.

The preoperative imaging should be conducted with magnetic resonance imaging (MRI), and should consist of multisequence acquisitions permitting analysis with established morphometric approaches. ${ }^{36}$ The collection of high-resolution, three-dimensional (3-D) T1weighted, 2-D proton density-weighted, and 2-D T2weighted images preoperatively should be accomplished according to standards recommended by the International Consortium for Human Brain mapping. As an example, on a GE 1.5T Signa series MR scanner an axial 3-D RF-spoiled gradient echo sequence includes: IR prep; $\mathrm{TE}=5 \mathrm{msec}$; $\mathrm{TI}=300 \mathrm{msec}$; flip angle $=20$ degrees; $16 \mathrm{kHz}$ bandwidth; $256 \times 160$ matrix; 1 excitation; field-of-view, $38 \mathrm{~cm}$; and $1.5 \mathrm{~mm}$ slice thickness. This sequence can be acquired in less than 6 minutes and has the advantage that TS patients with active facial tics can be imaged several times ( 3 to 5 acquisitions in succession) to obtain at least one data set without the intrusion of movement artifacts. Image sets without significant motion artifact can be later summed for enhancement of signal-to-noise. In addition, axial fast spin-echo proton density and T2-weighted images should be obtained. An example pulse sequence for GE Signa 1.5T scanners includes the following: $\mathrm{TE}=12 \mathrm{msec}$ and 120 msec; TR $=4,000$ msec; 8 echo-train-length; $15.6 \mathrm{kHz}$ bandwidth; $256 \times 160$ matrix; field-of-view $38 \mathrm{~cm}$, slice thickness $1.5 \mathrm{~mm}$, using phase-correction and autoshimming options. The preoperative MRI should be normal without evidence of significant structural lesions.

Postoperative imaging should be performed after allowing sufficient time to eliminate transient postoperative changes. Experience with DBS for other movement disorders suggests that 1 month is sufficient for this. Although proton MRI has been performed safely in patients with the DBS stimulation amplitudes set to zero (the MR will toggle the stimulator on an off, but no current will be deposited), precautions must be taken that the DBS leads are not positioned so as to result in inductive heating during imaging ${ }^{37}$ (contact the DBS manufacturer for further details and recommendations on postoperative use of MR). Safety precautions require in addition that all patients be warned of the dangers of exposure to diathermy devices.

Recent case reports of untoward effects of MRI in subjects with implanted DBS systems suggests that Xray computed tomography (CT), rather than MRI, may be the most prudent approach to postoperative verification of lead placement. A steep axial (Towne's view, to avoid unnecessary ocular irradiation) slice orientation with 1.25 - to $1.50-\mathrm{mm}$-thick contiguous slices should be obtained to extend from the anterior horns of the lateral ventricles through the parieto-occipital cerebral cortex. Dosage technique should be used such that reconstructions adequately depict electrode contacts without excessive beam-hardening artifact (e.g., $170 \mathrm{KeV}, 140 \mathrm{MS}$ 
technique in a GE Lightspeed scanner). The postoperative CT can be digitally coregistered with the preoperative MRI to improve anatomic localization of the electrode contacts. ${ }^{36,38}$

\section{POSTOPERATIVE ASSESSMENT}

Experience with DBS in Parkinson's disease and tremor has shown that there is often a "microlesion" effect shortly after electrode placement. , $39,40^{\text {In dystonia, }}$ it may take several days to weeks for the effect of stimulation to become apparent. ${ }^{41}$ Thus, to evaluate DBS in TS, it is important that the postoperative assessment of efficacy and side effects be done after any "microlesion" effect has subsided and after the stimulation settings have been stable for several weeks. These should be serial, blinded evaluations. At least 24 hours should elapse between OFF and ON evaluations, and both patient and examiner should be blind to whether the stimulation is on or off. Once the typical latent period between stimulation onset and optimal tic reduction has been established, the time between ON and OFF testing may be modified. A comprehensive preoperative assessment should be performed within 1 month of the surgery. A reasonable schedule for postoperative ON/OFF evaluations would be 3,6 , and 12 months after surgery. We recommend that annual ON/OFF evaluation be performed thereafter to provide information on long-term effects, It is important to note that, before reaching the stage at which these evaluations can be scheduled, several empiric adjustments to the stimulation parameters may be necessary to optimize the stimulation results. Again, there is little experience with this procedure in TS. Accordingly, we recommend that only centers with extensive experience programming DBS take on this responsibility.

Tics should be evaluated with two methods. The YGTSS is the most commonly used scale and has good validity and reliability. However, it is subjective and based on patient (and family) report. Therefore, a blinded video-based rating should also be performed. ${ }^{26}$ The potential for sampling error can limit both the YGTSS and video-based ratings.

There should be careful pre- and postoperative assessment of comorbid symptoms with valid and reliable instruments. We do not intend to endorse specific instruments but, rather, encourage the use of these tools to ensure uniformity across centers. For assessment of obsessive-compulsive symptoms, the Yale-Brown Obsessive Compulsive Scale symptom checklist and severity scale should be used. ${ }^{42,43}$ For evaluation of ADHD symptoms, the Conners' Adult ADHD Rating Scale should be used. ${ }^{44}$
Comprehensive neurological and psychiatric examinations should be performed at each visit.

It is essential that a careful neuropsychological evaluation be performed. These should be performed before surgery and again 6 and 12 months after surgery with stimulators on and again with stimulators off. The potential side effects of DBS of CM-PF thalamus, anterior $\mathrm{GPi}$, and anterior limb of the internal capsule are not well defined. Thus, early investigations must carefully explore for untoward effects under all stimulation conditions.

The accumulated body of scientific evidence on the neurocognitive deficits in TS have yielded relatively consistent findings with respect to intellectual ability and the presence of specific neuropsychological deficits and learning disorders. ${ }^{45}$ Problems in these areas are present in a significant percentage of patients. The literature to date has suggested that intellectual ability is normally distributed in TS. Whether or not individuals with TS have significant discrepancies between their verbal and nonverbal abilities remains unclear due to the lack of longitudinal studies. Cross-sectional studies have suggested that, with aging, TS patients may develop a drift between their verbal and performance IQ with decrement in the performance IQ and relative stability in the verbal IQ. This change suggests the possibility of greater nondominant than dominant hemispheric dysfunction in TS.

The prevalence of learning disabilities in TS appears to be similar to the base rates reported for the general population. There is evidence to suggest that the prevalence of learning disorders in TS may actually be lower than the general population when strict diagnostic criteria are applied. When learning disabilities are present in TS patients, they tend to be specific for difficulties in mathematics and written language. Specific neuropsychological deficits in TS consistently have been reported to include visuomotor integration problems, impaired fine motor skills, and executive dysfunction, although recent studies have suggested that the presence of ADHD may more accurately explain executive function (EF) deficits in TS.

Moreover, the presence of comorbid neuropsychiatric conditions in TS, notably ADHD and OCD, appear to increase significantly the likelihood that an individual with TS will also have intellectual deficits, a learning disability, or some demonstrable cognitive impairment. Thus, the role of neuropsychological assessment is to identify specific cognitive deficits that might be present in TS and that might be affected by DBS. 


\section{POTENTIAL NEUROPSYCHOLOGICAL TEST BATTERY FOR USE IN DBS TRIALS}

To monitor for potential adverse neuropsychological effects of DBS, a minimum test battery is recommended, using tests that are brief, easy to administer, and do not require a formally trained neuropsychologist (total administration time is approximately 45 minutes). These cognitive tests all have established psychometric properties; some have alternate forms for repeated testing and have demonstrated usefulness in TS samples.

\section{Grooved Pegboard}

This test is a timed motor speed and dexterity task that requires pegs to be placed in grooved holes, using each hand independently. Performance on tasks of fine motor skill highly dependent on visual perceptual skills, such as the Grooved Pegboard, appears to be consistently impaired in TS patient samples ${ }^{46-48}$ Administration time is approximately 5 minutes.

\section{Judgment of Line Orientation}

This is a motor-free test of visuospatial organization that requires the observer to judge the orientation of lines in space. In a recent study by Sheppard and colleagues ${ }^{49}$ using a line orientation task, TS subjects were shown to be right-biased in judging the midpoint of horizontal. Administration time is approximately 10 minutes.

\section{Trailmaking Test, Forms A and B}

This test is a timed, paper and pencil measure of EF involving numeric (A) and then alternating alphanumeric (B) sequencing. This visuomotor task requires mental tracking, sequencing, and set-shifting. ${ }^{51}$ Across various measures of EF in TS studies, a consistent finding has emerged on measures that may rely more heavily on timed tasks involving visuomotor skill ${ }^{52}$ such as Trailmaking. Administration time is approximately 5 minutes.

\section{The Hopkins Verbal Learning Test}

This test assesses verbal learning and memory by using a common memory task requiring the participant to recall a list of words that is repeated multiple times. Short delay-free recall, long delay-free recall, cued short and long recall, and intrusion errors can be measured with this test. Mahone and colleagues ${ }^{51}$ found that TS patients demonstrated significantly more intrusion errors on verbal list learning trials than controls. Administration time is approximately 15 minutes.

\section{Verbal Fluency (COWALT)}

This is a verbal test that measures word production. The participant is asked to produce as many words as possible beginning with a given letter (F-A-S) in a limited period of time, which is 60 seconds per letter. ${ }^{53}$ In a study by Brand and associates ${ }^{54}$ that used a measure of verbal fluency in children with TS alone and TS plus ADHD, the TS plus ADHD group performed significantly worse on the verbal fluency task. Administration time is approximately 5 minutes.

\section{SUMMARY}

Deep brain stimulation has the potential to be an effective therapy in a carefully selected subset of adult patients with TS in which tics are severe and intractable to medical therapy. However, at this time, there are many unknowns about the potential application of this therapy. Initial investigations must use rigorous inclusion and exclusion criteria, pre- and postoperative assessment using standardized rating scales by experts in TS and comorbid psychiatric disorders, careful documentation of electrode location, and comprehensive postoperative assessments including neurological, psychiatric, and neuropsychological evaluations. Each investigating site should use the same anatomical nomenclature, assessment protocols, and outcome instruments so that results can be compared across sites. Ideally, DBS will be performed at centers with expertise in diagnosing and treating TS in a multidisciplinary manner. Recognizing the need for a timely exchange and on-going assessment of clinical experience among those in the field who are exploring the DBS option with TS patients, the TSA is pursuing currently an initiative to facilitate the establishment of a database repository that would be available to the community.

\section{REFERENCES}

1. Rosenow JM, Mogilnert AY, Ahmed A, Rezai AR. Deep brain stimulation for movement disorders. Neurol Res 2004;26:9-20.

2. Lyons KE, Pahwa R. Deep brain stimulation and essential tremor. J Clin Neurophysiol 2004;21:2-5.

3. Lozano AM, Mahant N. Deep brain stimulation surgery for Parkinson's disease: mechanisms and consequences. Parkinsonism Relat Disord 2004;10(Suppl. 1):S49-S57.

4. Vonck K, Boon P, Goossens L, et al. Neurostimulation for refractory epilepsy. Acta Neurol Belg 2003;103:213-217.

5. Greenberg BD, Price LH, Rauch SL, et al. Neurosurgery for intractable obsessive-compulsive disorder and depression: critical issues. Neurosurg Clin N Am 2003;14:199-212.

6. Diederich NJ, Kalteis K, Stamenkovic M, Pieri V, Alesch F. Efficient internal pallidal stimulation in Gilles de la Tourette syndrome: a case report. Mov Disord 2005;20:1496-1499.

7. Flaherty AW, Williams ZM, Amirnovin R, et al. Deep brain stimulation of the anterior internal capsule for the treatment of Tourette syndrome: technical case report. Neurosurgery 2005; 57(Suppl.):E403; discussion E403.

8. Houeto JL, Karachi C, Mallet L, et al. Tourette's syndrome and deep brain stimulation. J Neurol Neurosurg Psychiatry 2005;76: 992-995.

9. Temel Y, Visser-Vandewalle V. Surgery in Tourette syndrome. Mov Disord 2004;19:3-14.

10. Visser-Vandewalle V, Temel Y, Boon P, et al. Chronic bilateral thalamic stimulation: a new therapeutic approach in intractable 
Tourette syndrome. Report of three cases. J Neurosurg 2003;99: 1094-1100.

11. Leckman JF. Tourette's syndrome. Lancet 2002;360:1577-1586.

12. Defer GL, Widner H, Marie RM, Remy P, Levivier M. Core assessment program for surgical interventional therapies in Parkinson's disease (CAPSIT-PD). Mov Disord 1999;14:572-584.

13. Quinn N, Brown R, Craufurd D, et al. Core Assessment Program for Intracerebral Transplantation in Huntington's Disease (CAPITHD). Mov Disord 1996;11:143-150.

14. Leckman J, Cohen D. Tourette's Syndrome-Tics, Obsessions, Compulsions: Developmental Psychopathology and Clinical Care. New York: John Wiley and Sons; 1999.

15. Dooley JM, Stokes A, Gordon KE. Pseudo-tics in Tourette syndrome. J Child Neurol 1994;9:50-51.

16. Tan EK. Psychogenic tics: diagnostic value of the placebo test. J Child Neurol 2004;19:976-977.

17. Kurlan R, Deeley C, Como PG. Psychogenic movement disorder (pseudo-tics) in a patient with Tourette's syndrome. J Neuropsychiatry Clin Neurosci 1992;4:347-348.

18. Leckman J, Zhang H, Vitale A, et al. Course of tic severity in Tourette syndrome: the first two decades. Pediatrics 1998;102:14-19.

19. Erenberg G, Cruse R, Rothner A. The natural history of Tourette syndrome: a follow-up study. Ann Neurol 1987;22:383-385.

20. Robertson M. Tourette syndrome, associated conditions and the complexities of treatment. Brain 2000;123:425-462.

21. Jankovic J. Tourette's syndrome. N Engl J Med 2001;345:11841192.

22. Robertson MM, Stern JS. Gilles de la Tourette syndrome: symptomatic treatment based on evidence. Eur Child Adolesc Psychiatry 2000;9(Suppl. 1):I60-I75.

23. Singer HS. The treatment of tics. Curr Neurol Neurosci Rep 2001;1:195-202.

24. Piacentini J, Chang S. Habit reversal training for tic disorders in children and adolescents. Behav Modif 2005;29:803-822.

25. Wilhelm S, Deckersbach T, Coffey BJ, Bohne A, Peterson AL, Baer L. Habit reversal versus supportive psychotherapy for Tourette's disorder: a randomized controlled trial. Am J Psychiatry 2003;160:1175-1177.

26. Goetz CG, Pappert EJ, Louis ED, Raman R, Leurgans S. Advantages of a modified scoring method for the Rush Video-Based Tic Rating Scale. Mov Disord 1999;14:502-506.

27. Goetz CG, Tanner CM, Wilson RS, Shannon KM. A rating scale for Gilles de la Tourette's syndrome: description, reliability, and validity data. Neurology 1987;37:1542-1544.

28. Leckman JF, Riddle MA, Hardin MT, et al. The Yale Global Tic Severity Scale: initial testing of a clinician-rated scale of tic severity. J Am Acad Child Adolesc Psychiatry 1989;28:566-573.

29. Albin RL, Koeppe RA, Bohnen NI, et al. Increased ventral striatal monoaminergic innervation in Tourette syndrome. Neurology 2003;61:310-315.

30. Braun AR, Randolph C, Stoetter B, et al. The functional anatomy of Tourette's syndrome: an FDG-PET study. II. Relationships between regional cerebral metabolism and associated behavioral and cognitive features of the illness. Neuropsychopharmacology 1995;13:151-168.

31. Braun AR, Stoetter B, Randolph C, et al. The functional anatomy of Tourette's syndrome: an FDG-PET study. I. Regional changes in cerebral glucose metabolism differentiating patients and controls. Neuropsychopharmacology 1993;9:277-291.

32. Peterson B, Leckman JF. A functional magnetic resonance imaging study of tic suppression in Tourette syndrome. Arch Gen Psychiatry 1998;54:326-333.

33. Peterson B, Thomas P, Kane MJ, et al. Basal ganglia volumes in patients with Gilles de la Tourette syndrome. Arch Gen Psychiatry 2003;60:415-424
34. Alexander GE, DeLong MR, Strick PL. Parallel organization of functionally segregated circuits linking basal ganglia and cortex. Annu Rev Neurosci 1986;9:357-381.

35. Starr P, Christine C, Theodosopoulos $P$, et al. Implantation of deep brain stimulators into the subthalamic nucleus: technical approach and magnetic resonance imaging-verified lead locations. J Neurosurg 2002;97:370-387.

36. Mazziotta J, Toga A, Evans A, et al. A probabilistic atlas and reference system for the human brain: International Consortium for Brain Mapping (ICBM). Philos Trans R Soc Lond B 2001;356: 1293-1322.

37. Rezai AR, Finelli D, Nyenhuis JA, et al. Neurostimulation systems for deep brain stimulation: in vitro evaluation of magnetic resonance imaging-related heating at 1.5 Tesla. J Magn Reson Imaging 2002;15:241-250.

38. Meyer CR, Boes JL, Kim B, et al. Demonstration of accuracy and clinical versatility of mutual information for automatic multimodality image fusion using affine and thin-plane spline warped geometric deformations. Med Image Anal 1997;1:195-206.

39. Loher TJ, Gutbrod K, Fravi NL, Pohle T, Burgunder J-M, Krauss JK. Thalamic stimulation for tremor. Subtle changes in episodic memory are related to stimulation per se and not to a microthalamotomy effect. J Neurol 2003;250:707-713.

40. Obwegeser AA, Uitti RJ, Witte RJ, Lucas JA, Turk MF, Wharen RE Jr. Quantitative and qualitative outcome measures after thalamic deep brain stimulation to treat disabling tremors. Neurosurgery 2001;48:274-281; discussion 281-274.

41. Coubes P, Roubertie A, Vayssiere N, Hemm S, Echenne B. Treatment of DYT1-generalised dystonia by stimulation of the internal globus pallidus. Lancet 2000;355:2220-2221.

42. Goodman WK, Price LH, Rasmussen SA, et al. The Yale-Brown Obsessive Compulsive Scale. I. Development, use, and reliability. Arch Gen Psychiatry 1989;46:1006-1011.

43. Goodman WK, Price LH, Rasmussen SA, et al. The Yale-Brown Obsessive Compulsive Scale. II. Validity. Arch Gen Psychiatry 1989;46:1012-1016

44. Conners CK, Erhardt D, Sparrow E. CAARS. North Tonawanda, NY: Multi-Health Systems Inc; 1997.

45. Como PG. Neuropsychological function in Tourette syndrome Adv Neurol 2001:85:103-111.

46. Yeates KO, Bornstein RA. Attention deficit disorder and neuropsychological functioning in children with Tourette's syndrome. Neuropsychology 1994;8:65-74.

47. Bornstein RA. Neuropsychological correlates of obsessive characteristics in Tourette syndrome. J Neuropsychiatry Clin Neurosci 1991;3:157-162.

48. Bornstein RA. Neuropsychological functioning in Gilles de la Tourette's syndrome. Psychiatry Res 1990;33:73-81.

49. Sheppard DM, Bradshaw JL, Mattingly JB. Abnormal line bisection judgments in children with Tourette's syndrome. Neuropsychologia 2002;40:253-259.

50. Mink JW. Basal ganglia dysfunction in Tourette's syndrome: a new hypothesis. Pediatr Neurol 2001;25:190-198.

51. Mahone EM, Koth CW, Cutting L, Singer HS, Denckla MB. Executive function in fluency and recall measures among children with Tourette's syndrome for ADHD. J Int Neuropsychol Soc 2001;7:102-111.

52. Schultz RT, Carter AS, Scahill L, Leckman JF. Neuropsychological findings. In: Cohen DJ, editor. Tourette's Syndrome-Tics, Obsessions, Compulsion: Developmental Psychopathology and Clinical Care. New York: John Wiley and Sons; 1999. p 80-103.

53. Spreen O, Benton AL. Neurosensory Center Comprehensive Examination of Aphasia (NCCEA) - revised edition. Victoria: University of Victoria, Neuropsychology Laboratory; 1977.

54. Brand N, Geenee R, Oudenhoven M, et al. Brief report: cognitive functioning in children with Tourette's syndrome with and without co-morbid ADHD. J Pediatr Psychol 2002;27:203-208. 